\title{
Identification of a novel RYRI congenital laryngeal myopathy using laryngeal electromyography
}

Steven Frampton ${ }^{1}$, Eva Sorensen ${ }^{1}$, Asalet Yener ${ }^{1}$, Daniel Willdridge ${ }^{1}$, David Allen ${ }^{1}$, Marjorie Illingworth ${ }^{1}$, Catherine Mercer $^{1}$, Kate Heathcote ${ }^{2}$, Hasnaa Ismail-Koch ${ }^{1}$, Andrea Burgess ${ }^{1}$

\section{Introduction}

Congenital myopathies are non-dystrophic neuromuscular conditions presenting early in life with variable phenotype and severity. Mutations of Ryanodine receptor type I (RYRI) genes can result in congenital myopathy, but have not previously been documented to functionally compromise laryngeal function.
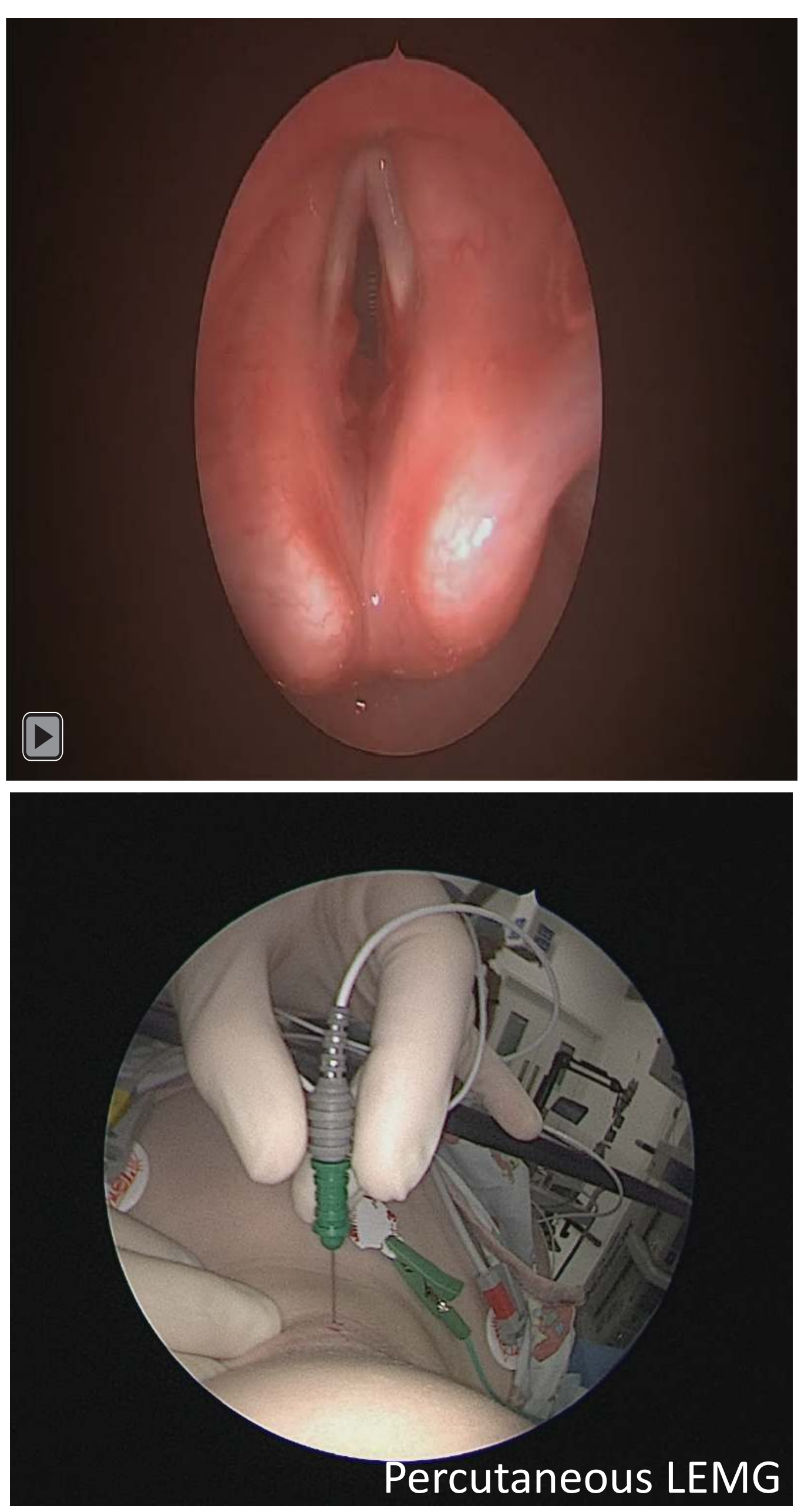

\section{Discussion}

LEMG findings consistent with laryngeal musculature involvement in RYR1 myopathy have not previously been reported. In this case, myopathic involvement of the larynx reinforced the decision to tracheostomise the patient following failed extubations. LEMG may be of use in future similar cases when the prognosis from a bilateral cord palsy would otherwise be unclear.

\section{Case}

A neonate was noted to have generalised hypotonia immediately after birth, particularly affecting the lower limbs, with respiratory distress syndrome and generalised hypotonia. He underwent echocardiogram, EEG and brain MRI which were all normal, but subsequent nerve conduction studies revealed evidence of a peripheral nerve or muscular disorder. He required nasogastric feeding and nocturnal BIPAP but returned home.

Chromosomal analysis (CGH) revealed a XYY karyotype but genetic testing for SMA and Prader-Willi syndrome were normal. Later, a thigh muscle biopsy provided features of mild congenital fibre type disproportion, and subsequent genetic testing revealed RYR1 mutations.

Following an episode of bronchiolitis he failed multiple extubation attempts and airway assessment with LEMG evidenced a bilateral vocal cord palsy with laryngeal myopathy consistent with that seen in his limb musculature.

The progress of RYRI myopathy is uncertain but the literature evidence suggests that the myopathy is not progressive and that individuals may make some mild improvement with time. Both parents were found to be RYRI carriers and were therefore at risk of Malignant Hyperthermia and were advised regarding this risk with future anaesthetics.
${ }^{1}$ Southampton Children's Hospital, University of Southampton NHS Foundation Trust, Southampton

2Poole Hospital NHS Foundation Trust, Poole

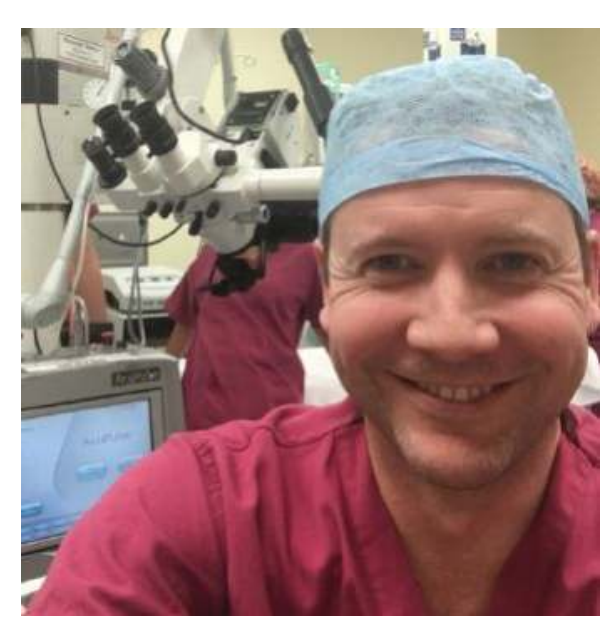

Poole Hospital WHS NHS Trust

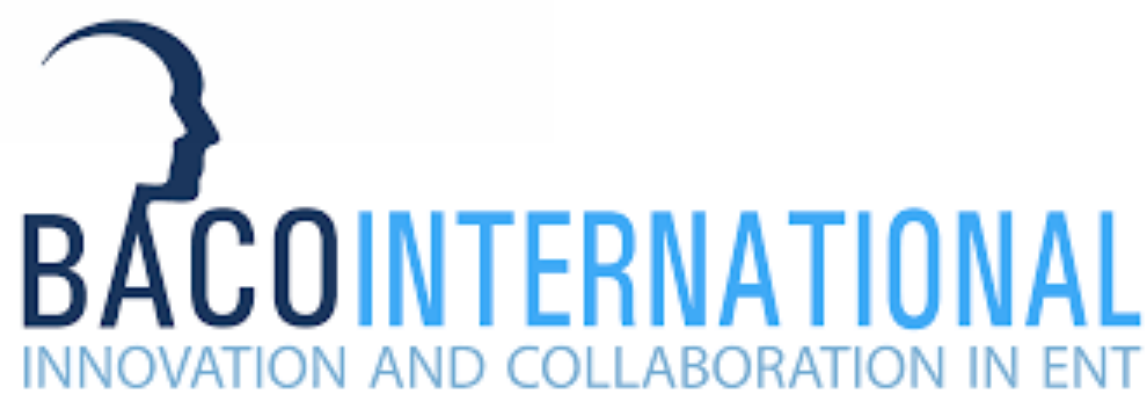

WHS University Hospital Southampton 